\title{
Comparison of Low-Mass and High-Mass Star Formation
}

\author{
Jonathan C. Tan \\ Depts. of Astronomy \& Physics, University of Florida, Gainesville, FL 32611, USA \\ email: jctan.astro@gmail.com
}

\begin{abstract}
I review theoretical models of star formation and how they apply across the stellar mass spectrum. Several distinct theories are under active study for massive star formation, especially Turbulent Core Accretion, Competitive Accretion and Protostellar Mergers, leading to distinct observational predictions. These include the types of initial conditions, the structure of infall envelopes, disks and outflows, and the relation of massive star formation to star cluster formation. Even for Core Accretion models, there are several major uncertainties related to the timescale of collapse, the relative importance of different processes for preventing fragmentation in massive cores, and the nature of disks and outflows. I end by discussing some recent observational results that are helping to improve our understanding of these processes.
\end{abstract}

\section{Introduction}

A universal theory of star formation that successfully describes the birth of low- and high-mass stars is a long-sought goal. In particular, large efforts have been made to see if massive stars form in a similar way to low-mass stars, i.e., via the Core Accretion paradigm, starting with pre-stellar cores (PSCs) that then collapse to form a single or binary protostar, with most matter accreting via a rotationally-supported disk that also helps to launch protostellar outflows (e.g., Shu et al. 1987; Inutsuka 2012). However, alternatives to this mechanism include Competitive Accretion (Bonnell et al. 2001; Wang et al. 2010) and Protostellar Mergers (Bonnell et al. 1998; Bally \& Zinnecker 2005; Moeckel \& Clarke 2011). Both have been proposed for massive star formation in crowded regions of protostellar clusters, the most common, perhaps near-universal, sites where massive stars form. However, these regions are also relevant for the bulk of star formation in galaxies and, in fact, Competitive Accretion has been invoked to explain most of the range of the stellar initial mass function $(\mathrm{IMF}) \gtrsim 1 M_{\odot}$ (e.g., Bate 2012). Here I discuss latest work that seeks to elucidate the formation mechanism of massive stars, including expected similarities and differences with low-mass protostars and role of environment.

\section{Massive Star Formation Environments}

Massive stars have dominated the universe since its earliest epochs. The first, "Pop III" stars, forming in individual dark matter minihalos from pure $\mathrm{H} \& \mathrm{He}$ gas, are thought to have been $\gtrsim 10-100 M_{\odot}$, so that they then initiated reionization and chemical enrichment of their surroundings (e.g., Bromm 2013). Continued radiative, mechanical and chemical feedback from massive stars is then theorized to have shaped the formation and evolution of galaxies (e.g., Vogelsberger et al. 2014). Observationally, the light seen from distant galaxies is dominated by that from massive stars (e.g., Sobral et al. 2015). This is true also in regions of nearby galaxies with active star formation, where the clustered nature of the process is readily apparent (e.g., Whitmore et al. 2014). There is a wide range of mass surface densities of these young star clusters from $\Sigma \sim 0.1-30 \mathrm{~g} \mathrm{~cm}^{-2}$ 
and a narrower range of sizes of $\sim 1-10$ pc (Tan et al. 2014 [T14]). Driven by these star clusters and dissolving OB associations, feedback on the interstellar medium is powered via radiation pressure, stellar winds, ionization and supernovae. Such feedback is likely to play a prominent role in the regulation of star formation rates (e.g., Walch et al. 2015).

Large fractions of all stars, including massive stars, form in clusters (e.g., de Wit et al. 2005; Gutermuth et al. 2009) and the distribution of star formation is much more clustered than that of molecular gas. This means that most stars and planetary systems may have been strongly influenced by massive star feedback at the time of their birth.

Another environment where massive stars form is galactic center regions, including in dense circumnuclear starburst disks, where mean disk mass surface densities can range from $\Sigma \sim 0.1-100 \mathrm{~g} \mathrm{~cm}^{-2}$ (Downes \& Solomon 1998; Wilson et al. 2014). Our Galactic center has a large population of young $(\lesssim 6 \mathrm{Myr})$, relatively massive stars, some orbiting in a disk-like structure around the supermassive black hole (e.g., Lu et al. 2014). The growth of supermassive black holes may be regulated by such star formation activity.

\section{The Physics of Star Formation}

The physics of star formation involves a competition between self-gravity of gas clumps (i.e., structures that fragment into star clusters) and cores (i.e., structures that collapse to a central rotationally-supported disk that forms a single star or small- $N$ multiple) and processes that resist collapse, i.e., various kinds of pressure forces, such as thermal, turbulent and magnetic. The evolution of these pressures needs to be followed by considering heating and cooling processes, generation and decay of turbulence, and generation and diffusion of $B$-fields. Once the clump or core contains stars, then support against collapse may be provided by mechanical feedback (i.e., protostellar outflows or stellar winds), radiation pressure (acting mostly on dust), or enhanced thermal pressure from photoionized regions. The chemical evolution of the gas and dust needs to be followed during collapse, especially as this helps set the trace ionization fraction that is important for coupling $B$-fields to the mostly neutral gas. Rotational support becomes important in protostellar disks, but theoretical prediction of their scale is quite uncertain due to the dominant role of magnetic braking in transferring angular momentum ( $\mathrm{Li}$ et al. 2014). Fragmentation of gas in the clump and in the core's disk will depend on the evolution of the local pressure and rotation/shear support contributions. To accurately model star formation, a wide range of spatial and temporal scales extending down to those of the protostellar surface need to be followed. Unlike the cosmological Pop III case, numerical simulations of "local" star formation face the additional challenge of uncertain choices for initial conditions: e.g., how close is the initial clump to virial and pressure equilibrium?

Due to the complexity of this highly nonlinear and high dynamic range problem, which remains beyond full computational tractability, there are many open questions. What causes a certain region of molecular cloud to form stars, i.e., does it typically occur due to an external trigger (e.g., converging flows, cloud collisions or stellar feedback) or via spontaneous gravitationally instability (e.g., as a cloud evolves and loses its earlier level of internal pressure support)? This question also relates to the specification of the initial conditions. Then, what is the accretion mechanism of the protostar, i.e., is most of the mass already organized into a self-gravitating PSC or is it later accreted competitively to the protostar from a previously unbound state from the larger-scale clump? Are protostellar mergers important? Are protostellar interactions that may disturb cores and disks important? What is the timescale of individual star formation: e.g., does a core collapse on a timescale that is similar to or much slower than the local free-fall time (for convenience defined with reference to that of a uniform density sphere: $\left.t_{\mathrm{ff}} \equiv(3 \pi /[32 G \rho])^{1 / 2}\right)$ ? 
Similarly, is the clump undergoing rapid global collapse to form a star cluster (Elmegreen 2000; Hartmann \& Burkert 2007) or is the process much slower allowing the clump to be in quasi equilibrium (Tan et al. 2006), perhaps regulated by outflow-driven turbulence (Nakamura \& Li 2014)? A consequence of these different possibilities is the range of age spreads present in young star clusters, again relative to the local clump free-fall time (Da Rio et al. 2014). Are massive stars the first to start forming in a cluster, becoming massive because of this head start (Wang et al. 2010; Bate 2012), or are their individual formation times short compared to the duration of cluster formation (McKee \& Tan 2002), allowing contemporaneous formation with lower-mass stars that is potentially independent of the evolutionary stage of the protocluster? What processes shape the stellar IMF and initial binary properties? How do these quantities vary with environment?

\subsection{Turbulent Core Accretion}

A variety of Core Accretion models for massive star formation have been proposed, extending the basic concepts of low-mass star formation models (see T14 for a review). The Turbulent Core Accretion model (McKee \& Tan 2003 [MT03]) recognizes that thermal pressure is unimportant for supporting massive PSCs (i.e., they are much more massive than the local Jeans or Bonnor-Ebert mass), so such support must come from some combination of nonthermal means, i.e., turbulence and/or magnetic fields. The next key assumption is that the pressure of the clump environment, which is $P_{\mathrm{cl}} \simeq G \Sigma_{\mathrm{cl}}^{2}$ for a self-gravitating cloud, sets the surface boundary condition of the core of a given mass. PSCs are modeled as singular polytropic spheres not too far from virial and hydrostatic equilibrium. Then the radius is given by $R_{c}=0.057 M_{c, 60}^{1 / 2} \Sigma_{\mathrm{cl}, 1}^{-1 / 2} \mathrm{pc}$, where $M_{c, 60}$ is the core mass normalized by $60 M_{\odot}$ and $\Sigma_{\mathrm{cl}, 1}$ is the clump mass surface density normalized by $1 \mathrm{~g} \mathrm{~cm}^{-2}$. This is the degree to which the core mass must be concentrated in order to become gravitationally unstable. Note that no assumption has been made about whether the PSC has taken a short or long time to form from the clump, although achieving approximate pressure and virial equilibrium is expected to take at least $\sim 1 t_{\mathrm{ff}}$. In principle, it is possible to estimate the age of a given PSC via comparison of predictions of astrochemical models, especially of deuterated species (e.g., Kong et al. 2015a), with observed abundances (§4.1). MT03 assumed cores and clumps have an internal radial density gradient $\rho \propto r^{-k_{\rho}}$, with $k_{\rho} \simeq 1.5$ set empirically (and also consistent with later studies of Infrared Dark Clouds (IRDCs) clumps/cores; Butler \& Tan 2012 [BT12]).

The core is then assumed to undergo inside-out collapse at a rate comparable with that of local free-fall collapse, i.e., $\dot{m}_{* d}=9.3 \times 10^{-4} \epsilon_{* d}\left(M_{c, 60} \Sigma_{\mathrm{cl}, 1}\right)^{3 / 4}\left(M_{* d} / M_{c}\right)^{1 / 2} M_{\odot} \mathrm{yr}^{-1}$, where $\dot{m}_{* d}$ is the rate of increase of the mass of the protostar and its disk, $\epsilon_{* d}$ is the current efficiency of the infall rate with respect to uninhibited collapse (values $\sim 0.5$ are expected due to protostellar outflow feedback, e.g., Zhang et al. 2014 [ZTH14]) and $M_{* d}$ is the idealized collapsed mass supplied to the central disk in the no-feedback limit. Note, this accretion rate estimate can be applied to stars forming from cores of all masses, with model assumptions only beginning to break down close to the Bonner-Ebert mass, $M_{\mathrm{BE}}=$ $0.050(T / 20 \mathrm{~K})^{2} \Sigma_{\mathrm{cl}, 1}^{-1} M_{\odot}$. The timescale for star formation is $t_{* f}=1.3 \times 10^{5} M_{c, 60}^{1 / 4} \Sigma_{\mathrm{cl}, 1}^{-3 / 4} \mathrm{yr}$, which has a very weak dependence on core mass. This timescale is similar to the clump's free-fall time. Thus it is important to know if the clump is undergoing rapid, free-fall collapse, or whether star cluster formation is a slower, more drawn-out affair.

The question of what, if anything, prevents fragmentation of massive PSCs is crucial, since this physics may play a decisive role in shaping the IMF via the (pre-stellar) core mass function (PS)CMF. In the Turbulent Fragmentation model of Padoan \& Nordlund (2002), a power law spectrum of super-Alfvénic turbulence is assumed and the size of dense cores is associated with the thickness of post-shock gas, so cores are born in a very 
dynamic fashion. This model has been compared to numerical simulations by Padoan et al. (2007; see also Collins et al. 2011; Hennebelle et al. 2011), who point out the potential importance of $B$-fields in setting the CMF. Hennebelle \& Chabrier (2008) presented a theory of the CMF based on the Press-Schecter statistical formalism applied to supersonic turbulence. In their model, more massive cores derive more support from turbulence, but also require lower density initial conditions to help set their larger "turbulent Jeans mass," than lower-mass cores. Alternatively, Kunz \& Mouschovias (2009) proposed a dominant role for $B$-fields, with the CMF being set by ambipolar diffusion. Motivated by their studies of massive, cold IRDC cores that contain $\gtrsim 100 M_{\mathrm{BE}}$, BT12 proposed $B$-field support was the dominant mechanism inhibiting fragmentation, so that $M_{c} \simeq M_{B}=38\left(B_{c} / \mathrm{mG}\right)^{3}\left(n_{\mathrm{H}, \mathrm{c}} / 10^{6} \mathrm{~cm}^{-3}\right)^{-2} M_{\odot}$, the magnetic critical mass (Bertoldi \& McKee 1992). One should note that massive starless cores, like massive stars, will be rare objects within the clump, and this rarity may be set by being in the high $B$-field-strength tail of the distribution. Krumholz \& McKee (2008) proposed radiative heating from surrounding lower-mass protostars prevents fragmentation of massive PSCs, which requires high accretion luminosities and thus high $\dot{m}_{* d}$ and so high $\Sigma_{\mathrm{cl}}, \gtrsim 1 \mathrm{~g} \mathrm{~cm}^{-2}$. This model predicts massive star formation requires the presence of an already forming protocluster and that massive protostars would not form from cold, dense cores within IRDCs.

Many numerical simulations of the collapse of massive, turbulent "cores" have been carried out. Dobbs et al. (2005) presented hydrodynamics-only simulations, finding extensive fragmentation into stars with masses of only $\sim 0.1 M_{\odot}$, and thus argued for Competitive Accretion. Krumholz et al. (2007) included dust reprocessed radiative feedback that heated the gas, raised the Jeans mass and thus reduced fragmentation. These simulations also showed radiation pressure does not prevent the formation of massive stars, given the high optical depths of the accretion flows. The strong influence of $B$-fields is illustrated in the results of Peters et al. (2011), who simulated a $100 M_{\odot}$ core with a $10 \mu \mathrm{G}$ field that suffered extensive fragmentation, and those of Seifried et al. (2011) and Myers et al. (2013), who included $\sim \mathrm{mG} B$-fields and found very limited fragmentation.

The analytic MT03 Turbulent Core model of collapse of massive cores in the limit of no fragmentation has been coupled to semi-analytic models of accretion disk structure (Zhang \& Tan 2011), disk-wind protostellar outflows (Zhang et al. 2013b) and a 1D numerical protostellar evolution code (ZTH14). These models have been used as inputs for continuum radiative transfer simulations to predict multiwavelength images and spectral energy distributions (SEDs). Lower-mass cores were studied by Zhang \& Tan (2015). Effects of varying the main environmental variable, $\Sigma_{\mathrm{cl}}$, have been explored. Density and temperature structures around example low- \& high-mass protostars are shown in Fig. 1.

Model predictions include the strong rise of fluxes at $\lambda<100 \mu \mathrm{m}$ as protostars grow in mass and luminosity and open up outflow cavities. Intensity profiles along the outflow axis are typically very asymmetric at $\lambda \lesssim 40 \mu \mathrm{m}$, with the near-facing outflow cavity being much brighter than the far-facing one. This asymmetry decreases for wavelengths $\gtrsim 70 \mu \mathrm{m}$ at which the core envelope is becoming optically thin. Protostellar cores in high $\Sigma_{\mathrm{cl}}$, i.e., high pressure, environments are smaller, denser, have higher accretion rates and luminosities, and are thus warmer, than cores of the same mass in low $\Sigma_{\mathrm{cl}}$ regions. High-mass envelopes and disks have similar overall morphologies and densities as their lower-mass counterparts, but, given the more luminous central protostar, are warmer.

In general, the advantages of the ZTH14 models for massive star formation are that they include: detailed, self-consistent physical models for the core structure and protostellar evolution; gas, as well as dust, opacities; and core properties that respond to clump environment, so typical massive cores are much smaller and denser and collapse with higher accretion rates compared to those of other models (e.g., Indebetouw et al. 2006; 


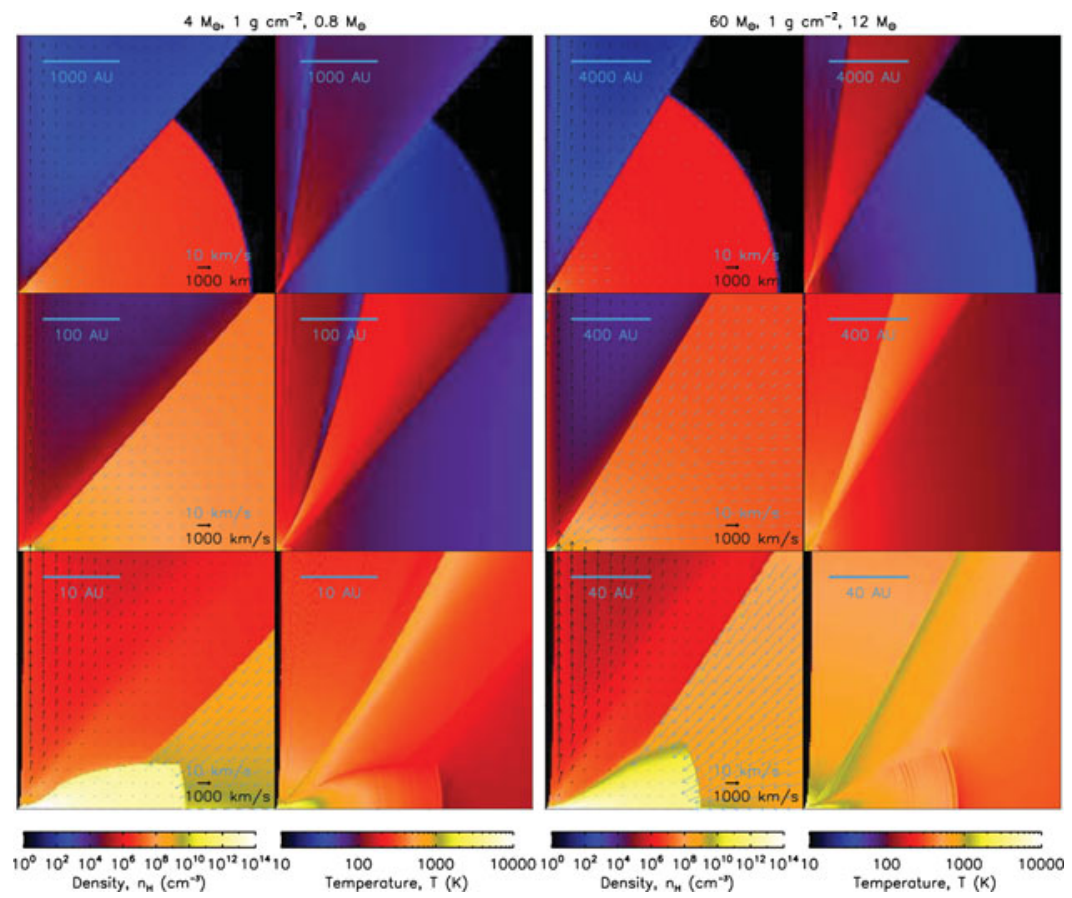

Figure 1. Comparison of low- (Zhang \& Tan 2015) and high-mass (ZTH14) protostars forming via Turbulent Core Accretion. Left 6 panels show slices through a $4 M_{\odot}$ core in a $1 \mathrm{~g} \mathrm{~cm}^{-2}$ clump that has collapsed to form a protostar with $0.8 M_{\odot}$ (i.e., $20 \%$ of initial core mass), which is located in the bottom left corner of each panel. Left column shows $\mathrm{H}$ nuclei number density, $n_{\mathrm{H}}$, and inflow/outflow velocities (note different arrow scales). Right column shows temperature. The view zooms from scale of the core (top row), to inner infall envelope (middle), to accretion disk (bottom). Right 6 panels show the equivalent figures for a $60 M_{\odot}$ core in the same clump environment that has collapsed to form a $12 M_{\odot}$ protostar (also $20 \%$ of initial $M_{c}$ ). Note linear scales in each row are $\simeq 4 \times$ larger than the low-mass case. While overall morphologies and absolute densities are quite similar, temperatures are significantly greater in the high-mass case, given its much larger luminosity of $5 \times 10^{4} L_{\odot}$ (cf., $300 L_{\odot}$ of the low-mass protostar).

Robitaille et al. 2006). However, they are still highly idealized in having: axisymmetric, smooth density structures (cf., Indebetouw et al. 2006); a simplified treatment of the interface between outflow cavity and infall envelope; and do not treat binary protostars.

An important difference of massive protostars compared to low-mass ones is that they can become significant sources of FUV and EUV radiation, since their accretion timescales are generally longer than their Kelvin-Helmholz contraction timescales. EUV photons should first lead to ionization of the protostellar outflows, i.e., initially in a phase of an "Outflow-Confined HII Region" (Tan \& McKee 2003; Tanaka et al. 2015). Here confinement is mostly in lateral directions, i.e., the disk and infall envelope are shielded. However, ionization soon becomes unconfined in directions along the outflow, which thus leads to a very elongated photoionized region that may appear as a cm continuum "radio jet." Later, the ionizing flux becomes strong enough to begin to have an effect on the accretion flow, e.g., by photoevaporation, although the presence of dust can significantly reduce the effectiveness of this feedback (Tanaka et al., in prep.), in contrast to the Population III case (McKee \& Tan 2008; Hosokawa et al. 2011). If massive protostars do not have strong, magnetocentrifugally-driven protostellar outflows, then their HII region structures may be "Accretion-Confined" (Keto 2007; Peters et al. 2010). 


\subsection{Competitive Accretion and Protostellar Mergers}

Competitive Accretion (Bonnell et al. 2001; Wang et al. 2010; Smith et al. 2011) involves protostars first forming from low-mass cores, with masses typically set by the BonnorEbert mass, i.e., $\ll 1 M_{\odot}$ in $\Sigma_{\mathrm{cl}} \sim 1 \mathrm{~g} \mathrm{~cm}^{-2}$ environments. The protostars then continue to accrete gas from the clump and for intermediate-mass and high-mass stars this clumpfed, Bondi-Hoyle mode of accretion plays the dominant role in setting the final mass.

A significant difference between Competitive Accretion and Turbulent Core Accretion is the former's much lower accretion rates to massive protostars if the clump is not undergoing rapid global collapse, i.e., if the star formation efficiency per free-fall time, $\epsilon_{\mathrm{ff}}$, of the protocluster is $\lesssim 0.1$. This is seen in the simulation of Wang et al. (2010), which includes outflow feedback that helps stabilize the clump. The most massive star in the simulation reaches $46.4 M_{\odot}$ in $1 \mathrm{Myr}$, with an average accretion rate of only $4.6 \times 10^{-5} M_{\odot} \mathrm{yr}^{-1}$. In absence of this feedback and in absence of $B$-fields that can help support massive PSCs, rapid global collapse and fragmentation of the clump is seen, leading to faster rates of Competitive Accretion (Bonnell et al. 2001; Smith et al. 2011).

For massive protostars forming via Competitive Accretion, the environment is required to be that near the center of a dense protocluster, which is also crowded with lower-mass protostars. Dynamical harassment of the protostar and its accretion envelope and disk is much more severe. This will limit the size of disks and lead to more rapid changes in their orientations, which will also be reflected in the orientations of any associated protostellar outflows. Simple bipolar outflows that maintain a fairly constant orientation would not be expected in these models, also because of presence of multiple overlapping, randomlyaligned outflows from the surrounding lower-mass protostars. Such morphologies are also expected to some extent for massive protostars forming via Turbulent Core Accretion due to turbulence in the core, small- $N$ multiple formation in the core, and neighboring protostars, so it is a question of the degree to which relatively ordered morphologies are preserved in the two scenarios, which still needs to be quantified in simulations.

Protostellar mergers (Bonnell et al. 1998), including via hardening of binaries (Bonnell \& Bate 2005) have been proposed as a massive star formation mechanism that operates in dense protocluster centers. For collisional growth to be rapid compared to cluster formation or massive stellar evolution timescales of $\sim 1-10 \mathrm{Myr}$, requires extreme stellar densities $\gtrsim 10^{8} \mathrm{pc}^{-3}$, equivalent to $n_{\mathrm{H}} \gtrsim 3 \times 10^{9} \mathrm{~cm}^{-3}$ (e.g., Moeckel \& Clarke 2011). Efficient growth by mergers leads to runaway growth of one or two extreme objects, rather than a smoothly filled upper IMF. For these reasons, mergers are generally considered to be unimportant in typical massive star-forming environments, although a merger has been invoked to explain activity of the Orion KL protostar (Bally \& Zinnecker 2005).

\section{Observational Constraints}

Here we discuss just a few recent examples of observations that test massive star formation theories and compare to low-mass cases (see T14 for a more extensive review).

\subsection{Pre-Stellar Cores}

One of the best studied "low-mass" PSCs is L1544 (Caselli \& Ceccarelli 2012). However, this core actually has $\sim 8 M_{\odot}$, and its slow, subsonic infall ( $\lesssim 10 \%$ of free-fall), suggests $B$-fields play a significant role in its dynamics (Keto et al. 2015).

Searches for more massive PSCs in higher $\Sigma_{\mathrm{cl}}$ environments have focussed on IRDCs. Tan et al. (2013), following up a sub-sample of the BT12 MIR extinction map peaks with $A L M A$, identified six cores via $\mathrm{N}_{2} \mathrm{D}^{+}(3-2)$ emission, with the most massive being $\mathrm{C} 1-\mathrm{S}$ with $\sim 60 M_{\odot}$. The observed line-widths are on average $80 \%$ of that predicted by the 
fiducial Turbulent Core model that assumes an Alfvén Mach number, $m_{A}=1$. However, for C1-S the observed velocity dispersion is only $40 \%$ of this level. Thus virial equilibrium would require a stronger $B$-field of $\sim 1 \mathrm{mG}$ (i.e., $m_{A}=0.3$ ). This field strength, given the core density of $n_{\mathrm{H}, \mathrm{c}} \sim 6 \times 10^{5} \mathrm{~cm}^{-3}$, also yields a magnetic critical mass close to C1-S's observed mass, which could help explain the core's limited fragmentation. Such $B$-field strengths are similar to those predicted using the empirical relation $B \simeq n_{\mathrm{H}}^{0.65} \mu \mathrm{G}$ (for $n_{\mathrm{H}}>300 \mathrm{~cm}^{-3}$ ) (Crutcher et al. 2010) and are also similar to values inferred in some massive protostars (§4.2). The deuteration fraction, $D_{\text {frac }}^{\mathrm{N} 2 \mathrm{H}+} \equiv\left[\mathrm{N}_{2} \mathrm{D}^{+}\right] /\left[\mathrm{N}_{2} \mathrm{H}^{+}\right]$, has been measured in C1-S to be $0.2-0.7$ (Kong et al. 2015b), several orders of magnitude greater than the cosmic $[\mathrm{D}] /[\mathrm{H}]$. By comparison with chemodynamical models, Kong et al. (2015b) conclude it is likely that C1-S is contracting at a relatively slow rate, $\sim 1 / 10$ th of free-fall, so as to have had enough time to reach this level of deuteration. This result needs confirmation by direct measurement of infall speeds, but this is challenging given the core is embedded in a much more massive and kinematically complex clump environment.

C1-S provides physical and chemical evidence for the existence of a massive, monolithic, centrally-concentrated, potentially-virialized pre-stellar or early-stage core. G11.92-0.61MM2 is another candidate (Cyganowski et al. 2014), but based only on mm continuum emission \& absence of line emission. The inferred mass of $\sim 30 M_{\odot}$ within $\sim 1000$ AU, i.e., $n_{\mathrm{H}} \gtrsim 10^{9} \mathrm{~cm}^{-3}$, yet without lines being seen from a lower density envelope, is surprising.

\subsection{Protostellar Cores}

Nearby low-mass protostellar cores are being studied in unprecedented detail with $A L M A$, including detection of rotating infall envelopes, bipolar outflows, and potentially Keplerian disks on scales $\lesssim 100 \mathrm{AU}$ (e.g., Codella et al. 2014). For the typically much more distant massive protostars, there are clear examples of collimated outflows (e.g., Beuther et al. 2002; Duarte-Cabral et al. 2013) and "rotating toroids" (e.g., Beltrán et al. 2011; Sánchez-Monge et al. 2013). Infall has been detected in a number of sources (e.g., Wyrowski et al. 2012). However, unambiguous detection of disks remains challenging, which is not unexpected if diameters are $\lesssim 1000 \mathrm{AU}$, i.e., $\lesssim 0.5$ " at $2 \mathrm{kpc}$. Girart et al. (2009) \& Q. Zhang et al. (2014) have inferred "hour-glass" morphologies of B-fields around some massive protostars, with field strengths $\sim 1 \mathrm{mG}$ and concluded they play a dynamically important role in massive star formation. Ionized, collimated outflows traced as radio continuum "jets" have been seen in some massive protostars (e.g., Gibb et al. 2003; Guzmán et al. 2014), although the relative importance of shock- versus photoionization remains to be established. The example of G35.20-0.74N contains all of the above elements and detailed radiative transfer models based on Turbulent Core Accretion have been fit to both its SED and resolved multiwavelength images that probe outflow axis intensity asymmetries to derive a protostellar mass of $m_{*} \sim 20-34 M_{\odot}$ embedded in a core with $M_{c} \simeq 240 M_{\odot}$ in a clump with $\Sigma_{\mathrm{cl}} \simeq 0.4-1 \mathrm{~g} \mathrm{~cm}^{-2}$ (Zhang et al. $2013 \mathrm{~b}$ ).

However, some massive protostellar cores appear much more disordered, with the most famous example being the Orion KL "hot core," which is thought to be powered by a massive protostar detected as radio "source I." There is an apparently "explosive" outflow from the region, which has been interpreted as a signature of a protostellar merger (Bally \& Zinnecker 2005). From X-ray images, Rivilla et al. (2013) inferred a protostellar density of $\sim 10^{6} \mathrm{pc}^{-3}$ in this region, still $\sim 100 \times$ less than that required for efficient mass growth by mergers $(\S 3.2)$. However, a single merger event involving a large disk-aided capture cross-section of $\sim 100$ AU may be expected to occur in $\lesssim 10^{5}$ yr. An alternative interpretation of Orion KL proposes that the explosive outflow is caused by tidal harassment of source I's accretion disk, leading to enhanced accretion and thus outflow, by a passing runaway star, the BN Object (Chatterjee \& Tan 2012). In this case, 
the interaction can be regarded as a moderate perturbation of the Core Accretion model. Future proper motion studies of source I and BN will help distinguish these scenarios.

\subsection{Protocluster Clumps}

Fragmentation of clumps and filaments is under active study (e.g., Beuther et al. 2013; Zhang et al. 2015), but interpretation of the detected structures is hampered by lack of observational probes of their $B$-fields. As reviewed by T14, infall times, $t_{\text {infall }} \equiv M_{\mathrm{cl}} / \dot{M}_{\text {infall }}$, relative to local free-fall time, $t_{\mathrm{ff}}$, have been measured in several clumps, including IRDCs, with typical observed ratios of $\sim 10$, indicating slow, quasi equilibrium collapse. This suggests a dynamically important role for $B$-fields, consistent with recent observations of two IRDCs by Pillai et al. (2015), and/or stabilization of collapse by protostellar outflow feedback, as modeled by Nakamura \& Li (2014). As discussed in $\S 3$, this question of the dynamical state of the star-forming clump environment, including nature of injected turbulence and distribution of $B$-field strengths, is crucial for its effect on fragmentation to the CMF and thus the formation mechanism of intermediate- and high-mass stars.

The quasi equilibrium cluster formation model (Tan et al. 2006) predicts age spreads of stars $\sim 10 t_{\mathrm{ff}}$ in systems that achieve final star formation efficiencies of $\sim 50 \%$, consistent with Da Rio et al.'s (2014) analysis of the Orion Nebula Cluster (ONC). It also implies there is significant time during star cluster formation for additional gas supply via infall from the clump surroundings and for dynamical evolution of the stellar population leading to mass segregation, so present-day locations of massive stars, such as $\theta^{1} C$ in the ONC, are not likely to have been where they formed. Improved observations of stellar kinematics (e.g., Foster et al. 2015; Cottaar et al. 2015) have the potential to test these theories.

\section{References}

Bally, J. \& Zinnecker, H. 2005, AJ, 129, 2281

Bate, M. R. 2012, MNRAS, 419, 3115

Bertoldi, F. \& McKee, C. F. 1992, ApJ, 395, 140

Beltrán, M. T. et al. 2011, A\&\&A, 525, 151

Beuther, H., Schilke P. et al. 2002, A\&A, 383, 892

Beuther, H., Linz, H., Tackenberg, J. et al. 2013, A\&SA, 553, 115

Bonnell, I. A. \& Bate, M. R. 2005, MNRAS, 362, 915

Bonnell, I. A., Bate, M. R., \& Zinnecker, H. 1998, MNRAS, 298, 93

Bonnell, I. A., Clarke, C. J., Bate, M. R., \& Pringle, J. E. 2001, MNRAS, 324, 573

Bromm V., 2013, Rep. Prog. Phys., 76, 11

Butler M. J. \& Tan J. C., 2012, ApJ, 754, 5 [BT12]

Caselli, P. \& Ceccarelli, C. 2012, A\&AR, 20, 56

Chatterjee, S. \& Tan, J. C. 2012, ApJ, 754, 152

Codella, C., Cabrit, S., Gueth, F. et al. 2014, A\&A, 568, L5

Collins, D., Padoan, P., Norman, M. L., \& Xu, H. 2011, ApJ, 731, 59

Cottaar, M., Covey, K. R., Foster, J. B. et al. 2015, ApJ, 807, 1

Crutcher, R. M., Wandelt, B., Heiles, C., et al. 2010, ApJ, 725, 466

Cyganowski, C. J., Brogan, C. L., Hunter, T. R. et al. ApJL, 796, 2

Da Rio, N., Tan, J. C., \& Jaehnig, K. 2014, ApJ, 795, 55

de Wit W. J., Testi L. et al. 2005, A\&SA, 437, 247

Dobbs, C. L. et al. 2005, MNRAS, 360, 2

Downes, D. \& Solomon, P. M. 1998, ApJ, 507, 615

Duarte-Cabral, A. et al. 2013, A\& A, 558, 125

Elmegreen, B. G. 2000, ApJ, 530, 277

Foster, J. B., Cottaar, M., Covey, K. R. et al. 2015, ApJ, 799, 136

Gibb, A. G. et al. 2003, MNRAS, 339, 198 
Girart, J. M., Beltrán, M. T., Zhang, Q. et al. 2009, Science, 324, 1408

Gutermuth, R. A., Megeath, S. T., Myers, P. C., et al. 2009, ApJS, 184, 18

Guzmán, A. E., Garay, G., Rodríguez, L. F., et al. 2014, ApJ, 796, 117

Hartmann, L. \& Burkert, A. 2007, ApJ, 654, 988

Hennebelle, P. \& Chabrier, G. 2008, ApJ, 684, 395

Hennebelle, P., Commerçon, B., Joos, M. et al. 2011, A\& A, 528, 72

Hosokawa, T., Omukai, K., Yoshida, N., \& Yorke, H. W. 2011, Science, 334, 1250

Indebetouw, R., Whitney, B. A., Johnson, K. E., \& Wood, K. 2006, ApJ, 636, 362

Inutsuka, S. 2012, PTEP, 2012, 01A307

Keto, E. 2007, ApJ, 666, 976

Keto, E., Caselli, P., \& Rawlings, J. 2015, ApJ, 446, 3731

Kong, S., Caselli, P., Tan, J. C. et al. 2015a, ApJ, 804, 98

Kong, S., Tan, J. C., Caselli, P. et al. 2015b, ApJ, submitted (arXiv:1509.08684)

Krumholz, M. R. \& McKee, C. F. 2008, Nature, 451, 1082

Krumholz, M. R., Klein, R. I. et al. 2007, ApJ, 656, 959

Kunz, M. W. \& Mouschovias, T. Ch. 2009, MNRAS, 399, L94

Li, Z.-Y., Banerjee, R. et al. 2014, Protostars 8 Planets VI, eds. Beuther et al., p173

Lu, J. R., Ghez, A. M., Morris, M. R. et al. 2014, IAUS, 303, 211

McKee C. F. \& Tan J. C., 2002, Nature, 416, 59

McKee C. F. \& Tan J. C., 2003, ApJ, 585, 850 [MT03]

McKee C. F. \& Tan J. C., 2008, ApJ, 681, 771

Myers A. T., McKee C. F. et al. 2013, ApJ, 766, 97

Moeckel, N. \& Clarke, C. J. 2011, MNRAS, 410, 2799

Nakamura, F. \& Li, Z.-Y. 2014, ApJ, 783, 115

Padoan, P. \& Nordlund, A. 2002, ApJ, 576, 870

Padoan, P., Nordlund, A., Kritsuk, A. G. et al. 2007, ApJ, 661, 972

Peters, T., Banerjee, R., Klessen, R. S., \& Mac Low, M.-M. 2011, ApJ, 729, 72

Peters, T., Mac-Low, M.-M., Banerjee, R. et al. 2010, ApJ, 719, 831

Pillai, T., Kauffmann, J., Tan, J. C. et al. 2015, ApJ, 799, 74

Rivilla, V., Martín-Pintado, J., Jiménez-Serra, I., \& Rodríguez-Franco, A. 2013, A\&A, 554, 48

Robitaille, T. P., Whitney, B. A., Indebetouw, R. et al. 2006, ApJS, 167, 256

Sánchez-Monge, Á., Cesaroni, R., Beltrán, M. T. et al. 2013, A\&̊A, L10

Seifried, D., Banerjee, R., Klessen R. S. et al. 2011, MNRAS, 417, 1054

Shu, F. H., Adams, F. C., \& Lizano, S. 1987, ARA\& $A$, 25, 23

Smith, R. J., Glover, S. C. O., Bonnell, I. A. et al. 2011, MNRAS, 411, 1354

Sobral, D., Matthee, J., Darvish, B. et al. 2015, ApJ, 808, 139

Tan, J. C., Beltrán, M. et al. 2014, Protostars 8 S Planets VI, eds. Beuther et al., p149 [T14]

Tan, J. C., Kong, S., Caselli, P. et al. 2013, ApJ, 779, 96

Tan, J. C., Krumholz, M. R., \& McKee, C. F. 2006, ApJL, 641, L121

Tan, J. C. \& McKee, C. F. 2003, astro-ph/0309139.

Tanaka, K. E. I., Tan, J. C., \& Zhang, Y. 2015, ApJ, submitted (arXiv:1509.06754)

Vogelsberger, M., Genel, S., Springel, V. et al. 2014, MNRAS, 444, 1518

Walch, S., Girichidis, P., Naab, T. et al. 2015, MNRAS, 454, 238

Wang, P., Li, Z.-Y., Abel, T., \& Nakamura, F. 2010, ApJ, 709, 27

Whitmore, B. C., Chandra, R., Bowers, A. S. et al. 2014, AJ, 147, 78

Wilson, C. D., Rangwala, N., Glenn, J. et al. 2014, ApJ, 789, 36

Wyrowski, F., Güsten, R., Menten, K. M. et al. 2012, A\&A, 542, L15

Zhang, Q., Qiu, K., Girart, J. M. et al. 2014, ApJ, 792, 116

Zhang, Q., Wang, K., Lu, X., \& Jiménez-Serra, I. 2015, ApJ, 804, 141

Zhang, Y. \& Tan, J. C. 2011, ApJ, 733, 55

Zhang, Y. \& Tan, J. C. 2015, ApJL, 802, L15

Zhang, Y., Tan, J. C., \& Hosokawa, T. 2014, ApJ, 788, 166 [ZTH14]

Zhang, Y., Tan, J. C., De Buizer et al. 2013b, ApJ, 767, 58

Zhang, Y., Tan, J. C. \& McKee 2013a, ApJ, 766, 86 